\title{
Nearby low-mass triple system GJ 795
}

\author{
E. V. Malogolovets, Yu. Yu. Balega, D. A. Rastegaev \\ Special Astrophysical Observatory, RAS, Nizhnii Arkhyz, Karachai-Cherkessian Republic, 357147 Russia \\ received:September 21, 2006/revised: November 24, 2006
}

\begin{abstract}
We report the results of our optical speckle-interferometric observations of the nearby triple system GJ 795 performed with the 6-m BTA telescope with diffraction-limited angular resolution. The three components of the system were optically resolved for the first time. Position measurements allowed us to determine the elements of the inner orbit of the triple system. We use the measured magnitude differences to estimate the absolute magnitudes and spectral types of the components of the triple: $M_{V}^{A a}=7.31 \pm 0.08, M_{V}^{A b}=8.66 \pm 0.10, M_{V}^{B}=8.42 \pm 0.10$, $S p_{A a} \approx \mathrm{K} 5, S p_{A b} \approx \mathrm{K} 9, S p_{B} \approx \mathrm{K} 8$. The total mass of the system is equal to $\Sigma \mathcal{M}_{A B}=1.69 \pm 0.27 \mathcal{M}_{\odot}$. We show GJ 795 to be a hierarchical triple system which satisfies the empirical stability criteria.
\end{abstract}

\section{INTRODUCTION}

According to the most recent concepts, stars form in small groups and clusters. The disruption of such groups results in the formation of both multiple systems and single stars. Stars in triple and more complex multiple systems make up for more than $20 \%$ of the Milky-Way population. The study of their dynamical and physical parameters is necessary for understanding the process of star formation as a whole. However, the now available observational data are insufficient for testing the theories of formation and evolution of multiple stars. We do not yet entirely understand the initial conditions and mechanisms of multiple star formation. The issues that still remain unclear include the conservation of angular momentum in the process of ' star formation; the dynamical stability of multiple systems with more than three stars; the effect of tidal interactions on the dynamical evolution of multiple systems; the distribution of orbital periods, eccentricities, component mass ratios, and correlations between these parameters; mutual orientations of the orbital planes in multiple systems, etc.

Of special interest is the study of multiple systems with low degree of hierarchy with comparable orbital periods and semimajor axes. The Orion Trapezium - a small cluster of very young and massive stars - is the most well known dynamically unstable multiple system. The disruption time scale of the Trapezium is estimated at $10^{4}-10^{6}$ years (Pflamm-Altenburg \& Kroupa, 2006). Main-sequence stars are also found in a number of systems that are potentially dynamically unstable (Fekel, 1981; Szebehely \& Zare, 1977, Tokovinin et al., 2005). The stability criteria for multiple stellar systems were analyzed by Eggleton \& Kiseleva, 1995. Golubev, 1967.

Send offprint requests to: E. V. Malogolovets, e-mail: evmag@sao.ru
Golubev, 1968: Harrington, 1972 $\quad$ Harrington, 1977, Steves \& Roy, 1999 and other authors. However, the conclusion about the dynamical instability of a particular system is often disproved when its orbital elements are refined. The triple system ADS 16904 with the periods of the inner and outer orbits equal to 15 and 150 years, respectively, is an example (Balega et al., 1999). According to all known criteria, the state of this system must be close to instability (Orlov \& Zhuchkov, 2005). However, new interferometric measurements including the data obtained with the 6-m telescope of the Special Astrophysical Observatory of the Russian Academy of Sciences indicate that the actual period of the outer orbit in this triple is twice longer and hence the system must be dynamically stable. So far, no systems with main-sequence components have been found that could be securely classified as dynamically unstable.

Known candidate objects with low orbit hierarchy, which therefore should be viewed as possible dynamically unstable multiple systems, include the nearby ( $d$ $\approx 16 \mathrm{pc}$ ) triple star GJ 795 (HD $196795=$ Hip 101955, $\alpha$ $=20^{h} 39^{m} 38^{s}, \delta=+04^{\circ} 58^{\prime} 19^{\prime \prime}$, epoch 2000.0). Its integrated spectral type corresponds to that of a K5V star. For decades, GJ 795 has been known as the visual pair Kui 99 (Kuiper, 1934) with a period of 40 years. During his spectroscopic survey of visual binaries with the CORAVEL radial-velocity scanner, Duquennoy (Duquennoy, 1987) found GJ 795 to contain a hitherto unknown spectroscopic subsystem with very low amplitude of radial-velocity variations. He concluded that the companion is bound to the main component of the binary and computed a preliminary model of the system by combining photometric and spectroscopic data with the computed orbital elements. At the same time, the inclination of the inner orbit of the triple was estimated based on the assumed component 
Table 1. Log of speckle observations

\begin{tabular}{c|c|c|c}
\hline Date & $\beta$ & $\mathrm{N}$ & $\begin{array}{c}\lambda / \Delta \lambda \\
\mathrm{nm}\end{array}$ \\
\hline 1998.7741 & 1 & 700 & $610 / 20$ \\
1999.8206 & 2 & 1500 & $610 / 20$ \\
2000.8752 & 1.5 & 1000 & $600 / 30$ \\
2001.7522 & 2 & 1500 & $545 / 30$ \\
& 2 & 1500 & $850 / 75$ \\
2002.7986 & $3-5$ & 1500 & $600 / 30$ \\
2003.9272 & 1 & 2000 & $545 / 30$ \\
& 1 & 2000 & $700 / 30$ \\
& 1 & 2000 & $800 / 110$ \\
2004.8232 & 1.5 & 2000 & $600 / 30$ \\
\hline
\end{tabular}

masses exclusively. The strongly inclined outer orbit also remained highly uncertain. To refine the pattern of component motions in GJ 795, this system was put in 1998 into the list of program stars for speckle-interferometric observations with the 6-m telescope of the Special Astrophysical Observatory of the Russian Academy of Sciences.

In this paper, we report the results of our speckleinterferometric observations of the relative measurements of components of GJ 795 and their differential photometry, and determine the parameters of orbital motion of stars and their dynamic masses. In conclusion, we discuss the dynamical stability of the system.

\section{OBSERVATIONS AND DATA ANALYSIS}

We performed speckle-interferometric observations of GJ 795 with the new facility mounted on the 6-m telescope of the Special Astrophysical Observatory of the Russian Academy of Sciences (Maximov et al., 2003). Its detector consists of a fast $1280 \times 1024$ Sony ICX085 CCD combined with a three-camera image-tube converter with electrostatic focusing. We recorded speckle-interferograms in the visible part of the spectrum with exposures ranging from 5 to 20 milliseconds. Table 1 lists the log of observations, which gives for each measurement the date of observation (as a fraction of Besselian year); seeing $\beta$ in arcsec; the number of speckle-interferograms in the series; filter parameter $\lambda / \Delta \lambda$ in $\mathrm{nm}$, where $\lambda$ and $\Delta \lambda$ are the central wavelength and half-bandwidth, respectively. We determined the relative component positions and magnitude differences from the power spectra of the speckle interferograms averaged over the series Balega et al., 2002a. The accuracy of the measured position parameters is $0.2-$ $4.0^{\circ}$ and 1-4 milliarcsec for position angle and angular separation, respectively. Measurement errors depend on a number of parameters: component separation, magnitude difference, and seeing $\beta$. The accuracy of the magnitude differences inferred from reconstructed power spectra depends on the same parameters. For objects in the $m_{V}=8-$ 10 magnitude interval it usually varies from 0.05 to 0.2 . We use bispectral analysis of the interferogram series to

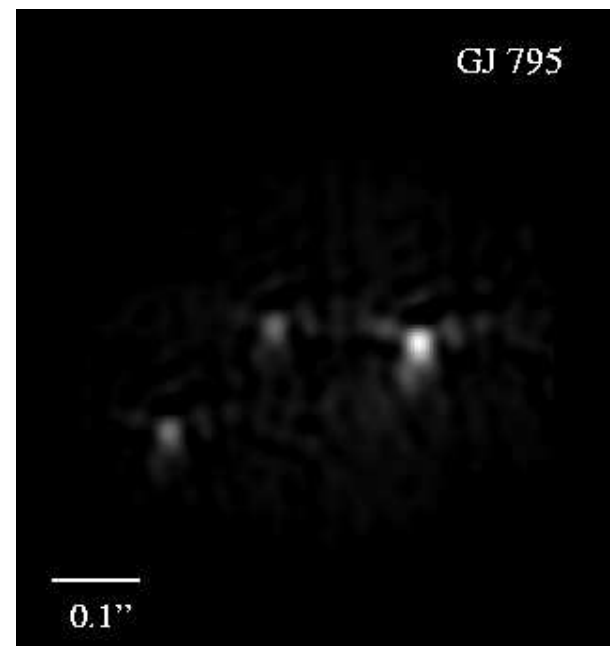

Fig. 1. The 610/20 nm image of GJ 795 (1998.77) reconstructed using bispectral analysis. Artefacts surrounding the point sources are due to various types. North is at the top and East on the left.

perform complete reconstruction - including that of modulus and phase — of the images (Lohmann et al., 1983. Weigelt, 1977). Figure 1 shows the reconstructed image of the triple star GJ 795 based on observations made in 1998.

\section{ABSOLUTE MAGNITUDES AND SPECTRAL TYPES}

As we already mentioned in the Introduction, Duquennoy (Duquennoy, 1987) carried out a detailed study of the radial-velocity variations of primary component $\mathrm{A}$ of the visual binary KUI 99 with the CORAVEL radial-velocity scanner. He detected no traces of the fainter star B in the spectra. On two nights in 1985, significant variations were observed in the profile of the correlation minimum that were due to the contribution of component $\mathrm{Ab}$ to the total flux. The resulting radial-velocity curve was used to determine the orbit of inner binary Aab with a period of $P=920.2$ days and eccentricity $e=0.747$. The preliminary model of the system, which included all three components, assumed a total mass and parallax of $\Sigma \mathcal{M}_{A B}=1.62 \pm 0.27 \mathcal{M}_{\odot}$ and $\pi=64 \pm 5$ mas, respectively. This model also made use of the empirical "mass - luminosity" relation for K6V (Aab) and K9V(B) type stars and a highly uncertain visual orbit of the outer pair $A B$ Baize, 1981.

According to Hipparcos (Perryman, 1997) data, the trigonometric parallax of GJ795 differs significantly from the above value $\left(\pi_{H i p}=53.82 \pm 2.21\right.$ mas $)$. However, Hipparcos trigonometric parallaxes for binary and multiple stars are known to be potentially fraught with extra errors due to wrong correction of component orbital motions in the process of Hipparcos data reduction (Balega et al., 2002b Shatskii \& Tokovinin, 1998). Soderhelm (Soderhjelm, 1999) corrected the parallax 
Table 2. Differential speckle interferometry of GJ 795

\begin{tabular}{c|l|c|c|c|c}
\hline $\begin{array}{c}\text { Date } \\
\text { BY }\end{array}$ & $\begin{array}{l}\text { Comp. } \\
\text { vector }\end{array}$ & $\Delta \mathrm{m}$ & $\sigma_{\Delta m}$ & $\begin{array}{c}\lambda / \Delta \lambda \\
\mathrm{nm}\end{array}$ & Reference \\
\hline 1998.7741 & Aa-Ab & 1.09 & 0.05 & $610 / 20$ & Balega et al. \\
& Aa-B & 0.88 & 0.05 & & $2002 \mathrm{a}$ \\
1999.8206 & Aa-Ab & 1.14 & 0.03 & $610 / 20$ & Balega et al. \\
& Aa-B & 0.94 & 0.03 & & 2004 \\
2000.8752 & Aa-Ab & 1.30 & 0.06 & $600 / 30$ & Balega et al. \\
& Aa-B & 1.02 & 0.06 & & 2006 \\
2001.7522 & Aa-Ab & 1.35 & 0.06 & $545 / 30$ & Balega et al. \\
& Aa-B & 1.11 & 0.06 & & 2006 \\
& Aa-Ab & 0.92 & 0.06 & $850 / 75$ & Balega et al. \\
& Aa-B & 0.68 & 0.06 & & 2006 \\
2004.8232 & Aa-Ab & 1.42 & 0.05 & $600 / 30$ & This paper \\
& Aa-B & 1.27 & 0.05 & & \\
\hline
\end{tabular}

for the effect of the orbital motion of the pair $\mathrm{AB}$ : $\pi_{H i p}^{*}=58.8 \pm 2.1$ mas. The corrected Hipparcos parallax agrees within the quoted errors with that given by Duquennoy (Duquennoy, 1987).

We performed differential speckle photometry of the system with the 6-m telescope of the Special Astrophysical Observatory of the Russian Academy of Sciences and list the results in Table 2. We set the $V$-band magnitude differences equal to $\Delta m_{A a A b}=1.35 \pm 0.06$ and $\Delta m_{A a B}=$ $1.11 \pm 0.06$, respectively, implying, given the corrected parallax, the absolute magnitudes of $M_{V}^{A a}=7.31 \pm 0.08$, $M_{V}^{A b}=8.66 \pm 0.10$, and $M_{V}^{B}=8.42 \pm 0.10$, respectively. These absolute magnitudes correspond to the spectral types of $S p_{A a} \approx \mathrm{K} 5, S p_{A b} \approx \mathrm{K} 9$, and $S p_{B} \approx \mathrm{K} 8$, respectively. The above spectral types agree well with the color index $B-V=1.24$ (Strassmeier et al., 2000). The space velocity components $(U, V, W)=(-75.5,-19.7,-42.3)$ (Strassmeier et al., 2000) and low emission level in the $\mathrm{H}$ and K Ca II lines (Gray et al., 2003) imply that this star should be classified as a Galactic-disk object with the age of 2-3 Gyr.

\section{ORBITAL PARAMETERS AND TOTAL MASSES}

The motion of components in a triple system can be subdivided into two components: the motion about the center of mass of the inner binary and the motion of the outer component about the common center of mass. The orbit of the outer system $\mathrm{AB}$ was computed by a number of authors from visual micrometric measurements (Baize, 1981; Heintz, 1984). Its main parameters are: a period $P$ of about 40 years; small eccentricity $e$, and high inclination with respect to the sky plane $\left(i \approx 85^{\circ}\right)$. Soderhjelm (Soderhjelm, 1999) refined the orbital elements of the pair $\mathrm{AB}$ by combining the data of ground-based observations with Hipparcos astrometry. He inferred a total mass of $\Sigma \mathcal{M}_{A B}=2.26 \pm 0.36 \mathcal{M}_{\odot}$, which exceeds significantly the mass estimated by Duquennoy (Duquennoy, 1987) based on spectroscopic and visual data. Despite the use of
Hipparcos astrometry, the outer orbit remains uncertain, mostly because of its high inclination.

We determined the preliminary orbital parameters of the subsystems of GJ 795 with allowance for new speckleinterferomeric measurements based on the Fourier transform of equations of motion (Monet, 1979). At the next stage, we refined the orbital elements via differential correction based on the least squares method (see comments in Forveille et al., 1999). We adopted early visual and several interferometric measurements from the Washington Catalog of Binary Stars (http://ad.usno.navy.mil/wds). We set the weights of speckle observations made with the 6-m telescope of the Special Astrophysical Observatory of the Russian Academy of Sciences to be 10 times higher than those of the visual and interferometric observations made with other telescopes. The main reason why we attributed such low weight factors to earlier data is that they did not allow for the binary nature of component $\mathrm{A}$, resulting in significant systematic errors.

We computed the orbit of the inner binary Aab based exclusively on the data of interferometric observations made with the 6-m telescope of the Special Astrophysical Observatory of the Russian Academy of Sciences. Seven measurements span over about 2.5 periods along the apparent ellipse of the orbit (Fig. 21). An attempt to use radial velocities from Duquennoy, 1987 to construct a combined orbit resulted in increased errors of the inferred orbital elements. This is due to the combined effect of small radial velocities of Aab system, small inclination of the inner orbit to the sky plane, and systematic errors in radialvelocity measurements due to the influence of the distant component B.

To convert the motion of component $\mathrm{B}$ to the centerof-mass frame of the inner binary Aab, we set the mass ratio equal to $q_{i n}=0.8$ based on the empirical "mass $M_{V}$ " relation of Henry \& McCarthy, 1993 and absolute magnitudes $M_{V}^{A a}=7.3$ and $M_{V}^{A b}=8.7$. We give the elements of the outer and inner orbits in Table 3 We list all position measurements made with the 6 -m telescope of the Special Astrophysical Observatory of the Russian Academy of Sciences and the corresponding residuals in Table 4 . The orbital parameters of the inner binary Aab agree well with those of the spectroscopic orbit by Duquennoy (Duquennoy, 1987) and those of the outer binary AB, with the refined orbit of Soderhelm (Soderhjelm, 1999). It is remarkable that the preliminary estimates of the inclination and semimajor axis of the inner orbit obtained by Duquennoy (Duquennoy, 1987) based on published empirical "mass - luminosity" relations coincided exactly with their true values inferred from the results of our interferometry.

Let us now determine the angle $\phi$ between the orbital planes:

$\cos \phi=\cos i_{\text {out }} \cos i_{\text {in }}++\sin i_{\text {out }} \sin i_{\text {in }} \cos \left(\Omega_{\text {out }}-\Omega_{\text {in }}\right),(1)$

where $i_{\text {out }}$ and $i_{\text {in }}$ are the tilt angles of the orbit of the outer and inner binaries with respect to the sky plane, respectively; $\Omega_{\text {out }}$ and $\Omega_{\text {in }}$ are the longitudes of the ascend- 


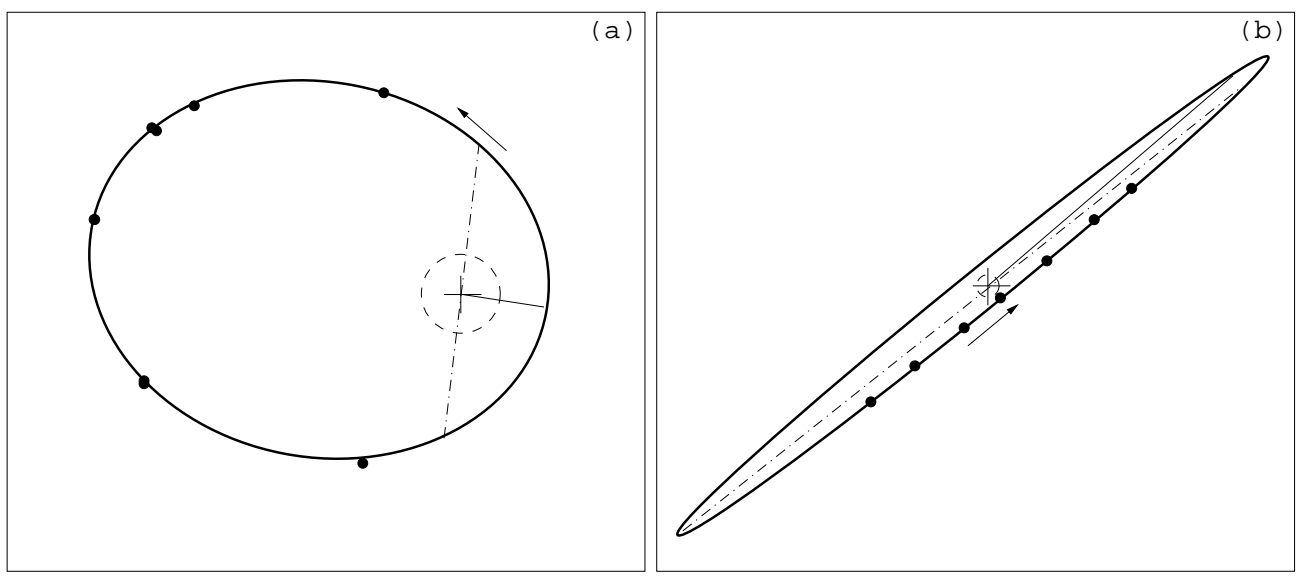

Fig. 2. Relative ellipses of the orbits of the triple system GJ 795: (a) orbit of binary Aab, (b) orbit of binary AB. The filled circles show the speckle-interferometric observations made with the 6-m telescope of the Special Astrophysical Observatory of the Russian Academy of Sciences; the solid line shows the position of the periastron, and the dottedand-dashed line, the line of nodes. The radius of the dashed circle is equal to 20 mas. Position parameters of component $\mathrm{B}$ are converted to the center-of-mass frame of binary Aab.

Table 3. Parameters of the inner and outer orbits in the triple system GJ 795

\begin{tabular}{l|l|l}
\hline & Aab & AB \\
\hline $\mathrm{P}$, years & $2.51 \pm 0.01$ & $39.4 \pm 0.2$ \\
$\mathrm{~T}$ & $2000.55 \pm 0.01$ & $1975.0 \pm 0.3$ \\
$\mathrm{e}$ & $0.620 \pm 0.006$ & $0.06 \pm 0.01$ \\
$\mathrm{a}$, mas & $120 \pm 2$ & $820 \pm 30$ \\
$\mathrm{i}^{\circ}$ & $18 \pm 3$ & $86.9 \pm 0.1$ \\
$\Omega^{\circ}$ & $174 \pm 11$ & $128.5 \pm 0.2$ \\
$\omega^{\circ}$ & $87 \pm 11$ & $212 \pm 2$ \\
$\sigma_{\theta}$ & 0.5 & 0.8 \\
$\sigma_{\rho}$ & 1 & 1 \\
\hline
\end{tabular}

ing node of the outer and inner binaries, respectively. We now use the angle values from Table 3 to obtain $\phi=74^{\circ}$.

The dynamic mass of the inner binaries as inferred from the orbital parameters and the corrected Hipparcos parallax of $\pi_{\text {Hip }}^{*}=58.8 \pm 2.1$ mas (Soderhjelm, 1999) is equal to $\Sigma \mathcal{M}_{A a b}=1.28 \pm 0.15 \mathcal{M}_{\odot}$. The mass of the entire system GJ 795 computed using the orbital parameters of binary $\mathrm{AB}$ is equal to $\sum \mathcal{M}_{A B}=1.69 \pm 0.27 \mathcal{M}_{\odot}$. According to Lang (Lang, 1992), the individual masses of stars in the system as inferred from their absolute magnitudes are equal to: $\mathcal{M}_{A a}=0.67 \mathcal{M}_{\odot}, \mathcal{M}_{A b}=0.57 \mathcal{M}_{\odot}, \mathcal{M}_{B}=0.54 \mathcal{M}_{\odot}$. The above estimates imply a mass of $\Sigma \mathcal{M}_{A a b}=1.24 \mathcal{M}_{\odot}$ for Aab binary and a total mass of $\sum \mathcal{M}_{A B}=1.78 \mathcal{M}_{\odot}$ for the entire system, which are consistent within the quoted errors with the total masses inferred using orbital parameters.

\section{HIERARCHY OF ORBITS AND THE KOZAI MECHANISM OF OSCILLATIONS}

The ratio of the orbital periods of the components of the system is equal to $P_{\text {out }} / P_{\text {in }}=15.7$, and hence the system is weakly hierarchical and its components form a single gravitationally bound system. Let us now try to estimate its dynamical stability using empirical criteria and criteria based on numerical simulations. According to one of such criteria suggested by Tokovinin (Tokovinin, 2004), the system is stable if the following inequality is satisfied:

$T=\frac{P_{\text {out }}\left(1-e_{\text {out }}\right)^{3}}{P_{\text {in }}}>T_{c}$,

where $P_{\text {out }}$ and $P_{\text {in }}$ are the orbital periods of the outer and inner binaries, respectively; $e_{\text {out }}$, the eccentricity of the orbit of the outer binary, and $T_{c}$ is the critical instability value, which is equal to 5 . The stability parameter for GJ 795 is equal to $\mathrm{T} \approx 13$ and hence the system is stable.

Based on numerical simulations, Harrington (Harrington, 1977) proposed the following stability parameter for triple systems, which depends on the ratios of the semimajor axes of the outer and inner orbits, $a_{\text {out }}$ and $a_{i n}$, and the eccentricity of the outer orbit:

$F=\frac{a_{\text {out }}\left(1-e_{\text {out }}\right)}{a_{\text {in }}}>F_{c}$.

Like in the case of empirical estimate, the value of this parameter, $F=6.42$, exceeds the critical level of $F_{c}=5.46$. However, these criteria should be used with much caution when applied to systems with orthogonal orbits.

The semimajor axes of the outer and inner binaries are equal to $a_{\text {out }} \approx 14 \mathrm{AU}$ and $a_{\text {in }} \approx 2 \mathrm{AU}$, respectively. The apoastron distance of the inner binary is equal to $\approx 3.2 \mathrm{AU}$. The eccentricity of the outer orbit is close to zero and therefore components of the triple are never at comparable distances from each other. 
Theoretical studies on the dynamics of multiple systems show that in the case of large angles between the orbital planes the inner and outer binaries (Kozai, 1962) exchange angular momentum. This mechanism triggers periodic variations (Kozai oscillations) of the eccentricity of the inner orbit, $e_{i n}$, and angle $\phi$ between the orbital planes. The quantity $\left(1-e_{i n}^{2}\right) \cos ^{2} \phi=$ const remains constant in this process. The following formula gives the period of Kozai cycle:

$P_{\text {kozai }} \sim P_{\text {out }}^{2} / P_{\text {in }}\left(1-e_{\text {out }}\right)^{3 / 2}$.

The period of Kozai oscillations for the triple star GJ 795 is equal to only 560 years. We may try to directly observe Kozai oscillations of the orbital parameters of binary Aab over several years of interferometric observations.

\section{CONSLUSIONS}

We use speckle-interferometric observations made in 19982004 with the 6-m telescope of the Special Astrophysical Observatory of the Russian Academy of Sciences to compute accurate visual orbits for the outer and inner binaries of the triple star GJ 795. This triple system belongs to the disk component of the Galaxy and is 2-3 Gyr old. Differential photometry of the components of this system made it possible to construct a complete model of GJ 795, which agrees well with modern empirical and theoretical relations. The absolute magnitudes of the components are equal to $M_{V}^{A a}=7.31 \pm 0.08, M_{V}^{A b}=8.66 \pm 0.10$, and $M_{V}^{B}=8.42 \pm 0.10$ and they correspond to the spectral types of $S p_{A a} \approx \mathrm{K} 5, S p_{A b} \approx \mathrm{K} 9$, and $S p_{B} \approx \mathrm{K} 8$, respectively.

The orbital periods are equal to 2.51 and 39.4 years for the inner and outer binaries, respectively. The angle between the planes of the inner and outer orbits is equal to $\phi=74^{\circ}$. The total dynamical masses $\Sigma \mathcal{M}_{A a b}=1.28 \pm 0.15 \mathcal{M}_{\odot}$ and $\sum \mathcal{M}_{A B}=1.69 \pm 0.27 \mathcal{M}_{\odot}$ are consistent with the estimated spectral types of the components.

We use the available empirical and theoretical stability criteria to conclude that GJ 795 is a gravitationally bound stable hierarchical system. For objects of this type, the Kozai mechanism should be efficient, which causes oscillations of the orbital eccentricities and the angle between the orbital planes. During its lifetime, the triple star GJ 795, whose Kozai period is equal to $P_{\text {kozai }} \approx 560$ years, must have undergone $\sim 10^{6}$ such periodic perturbations.

Acknowledgements. We are grateful to the night assistants at the 6-m telescope of the Special Astrophysical Observatory of the Russian Academy of Sciences for supporting the efficient work on the program. This work was supported by the Russian Foundation for Basic Research (grant no. 04-02-17563).

\section{References}

J. Pflamm-Altenburg and P. Kroupa, MNRAS (2006, in press). F. C. Fekel, Astrophys. J. 246, 879 (1981).
V. Szebehely, K. Zare, A\&A 58, 145 (1977).

A. Tokovinin, O. Kiyaeva, M. Sterzik, et al., A\&A 441, 695 (2005).

P. P. Eggleton and L. G. Kiseleva, Astrophys. J. 455, 640 (1995).

V. G. Golubev, Doklady Akad. Nauk SSSR 12, 259 (1967).

V. G. Golubev, Doklady Acad. Nauk SSSR 13, 373 (1968).

R. S. Harrington, Celest. Mech. 6, 322 (1972).

R. S. Harrington, AJ 82, 753 (1977).

R. Mardling and S. Aarseth, in Proceedings of the International Meeting on the dynamics of small bodies in the Solar system, a major key to Solar system studies, Ed. by B. A. Steves and A. E. Roy (Kluwer, Dordrecht, 1999), p. 385.

I. I. Balega, Yu. Yu. Balega, K.-H. Hofmann, et al., Astronomy Letters 25, 797 (1999).

V. V. Orlov and R. Ya. Zhuchkov, AZh 82, 3, 231 (2005).

G. P. Kuiper, PASP 46, 285 (1934).

A. Duquennoy, A\&A 178, 114 (1987).

A. F. Maximov, Y. Y. Balega, U. Beckman, et al., Bull. Spec. Astrophys. Obs. 56, 102 (2003).

I. I. Balega, Y. Y. Balega, K.-H. Hofmann, et al., A\&A 385, 87 (2002a).

A. W. Lohmann, G. Weigelt, and B. Wirnitzer, Appl. Opt. 22, 4028 (1983).

G. Weigelt, Opt. Commun. 21, 55 (1977).

P. Baize, A\&AS 44, 199 (1981).

M. A. C. Perryman, ESA, The Hipparcos and Tycho Catalogues (ESA Publ. Division, SP-1200, 1997).

Yu. Yu. Balega, A. A. Tokovinin, E. A. Pluzhnik, and G. Weigelt, Astronomy Letters 28, 773 (2002b).

N. I. Shatskii and A. A. Tokovinin, Astronomy Letters 24, 673 (1998).

S. Soderhjelm, A\&A 341, 121 (1999).

K. G. Strassmeier, A. Washuettl, T. Granzer, et al., A\&AS 142, 275 (2000).

R. O. Gray, C. J. Corbally, R. F. Garrison, et al., AJ 126, 2048 (2003).

W. D. Heintz, A\&AS 56, 5 (1984).

D. G. Monet, Astrophys. J. 234, 275 (1979).

T. Forveille, J.-L. Beuzit, X. Delfosse, et al., A\&A 351, 619 (1999).

B. D. Mason, G. L. Wycoff, and W. I. Hartkopf, http://ad.usno.navy.mil/wds

T. J. Henry and D. W. McCarthy, AJ 106, 773 (1993).

K. R. Lang, Astrophysical Data: Planets and Stars (SpringerVerlag, New York, 1992).

A. Tokovinin, in Rev. Mex. Astron. Astrof. Conf. Ser., Ed. by C. Allen and C. Scarfe (Instituto de Astronomia, UNAM, Mexico, 2004), Vol. 21, pp. 7-14.

Y. Kozai, AJ 67, 591 (1962).

I. I. Balega, Y. Y. Balega, A. F. Maksimov, et al., A\&A 422, 627 (2004).

I. I. Balega, Y. Y. Balega, A. F. Maksimov, et al., Bull. Spec. Astrophys. Obs. 59, 20 (2006). 
Table 4. Position parameters and residuals of the measurements of the triple system GJ 795

\begin{tabular}{c|l|c|c|c|c}
\hline Subsystem & Epoch & $\begin{array}{c}\theta \\
\text { degrees }\end{array}$ & $\begin{array}{c}\rho \\
\text { mas }\end{array}$ & $\begin{array}{c}(O-C)_{\theta} \\
\text { degrees }\end{array}$ & $\begin{array}{c}(O-C)_{\rho} \\
\text { mas }\end{array}$ \\
\hline Aab & 1998.7741 & 55.0 & 161 & 0.3 & -2 \\
& 1999.8206 & 105.7 & 164 & -0.5 & 1 \\
& 2000.8752 & 20.8 & 108 & 0.4 & 1 \\
& 2001.7522 & 78.2 & 185 & -0.2 & 0 \\
& 2001.7522 & 78.5 & 186 & 0.5 & 1 \\
& 2002.7986 & 150.3 & 98 & 0.9 & 2 \\
& 2003.9272 & 61.9 & 172 & -0.3 & -1 \\
& 2003.9272 & 61.9 & 174 & -0.3 & 1 \\
& 2003.9272 & 61.7 & 174 & -0.5 & 1 \\
& 2004.8232 & 105.2 & 164 & -0.1 & 0 \\
\hline AB & 1998.7741 & 135.4 & 358 & 0.2 & 2 \\
& 1999.8206 & 139.1 & 234 & -0.2 & 1 \\
& 2000.8752 & 153.2 & 108 & 0.0 & 1 \\
& 2001.7522 & 238.5 & 48 & 2.3 & 1 \\
& 2001.7522 & 236.8 & 45 & 0.6 & -2 \\
& 2002.7986 & 292.5 & 153 & 0.6 & 0 \\
& 2003.9272 & 300.3 & 286 & 0.1 & -3 \\
& 2003.9272 & 300.0 & 289 & -0.2 & 1 \\
& 2003.9272 & 299.8 & 289 & -0.4 & 1 \\
& 2004.8232 & 303.1 & 390 & 0.3 & -1 \\
\hline
\end{tabular}

